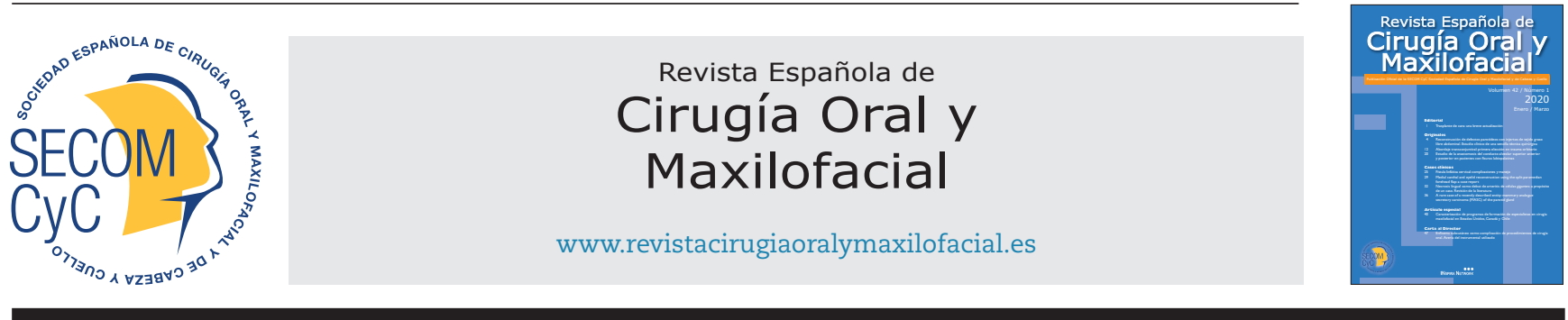

\title{
Original
}

\section{Reconstrucción de defectos parotídeos con injertos de tejido graso libre abdominal. Estudio clínico de una sencilla técnica quirúrgica}

\author{
Ignacio Arribas García ${ }^{a^{*}}$, Fátima Martínez Pérez ${ }^{b}$, Alberto García Hernández \\ Modesto Álvarez Flórez ${ }^{a}$
}

aServicio Cirugía Oral y Maxilofacial, Hospital Universitario de Canarias. Tenerife, España. 'Servicio Cirugía Oral y Maxilofacial, Hospital San Juan de Dios. Tenerife, España

\section{INFORMACIÓN DEL ARTÍCULO}

Historia del artículo:

Recibido el 25 de octubre de 2019

Aceptado el 3 de diciembre de 2019

\section{Palabras clave:}

Glándula parótida, parotidectomía, reconstrucción parotídea, injerto graso, estética facial, cirugía reconstructiva facial, relleno facial, tumor parotídeo.

\section{R E S U M E N}

Introducción: La cirugía parotídea es una técnica muy habitual y presenta múltiples tipos de abordajes y métodos de reconstrucción. Las distintas opciones de reconstrucción primarias empleadas son el colgajo del sistema músculo aponeurótico superficial (SMAS), la rotación del músculo esternocleidomastoideo (ECM), injertos libres/microvascularizados o la utilización de biomateriales tipo Alloderm, entre otros.

Se presenta nuestra experiencia en la utilización de injerto de grasa libre abdominal periumbilical para la reconstrucción primaria del defecto parotídeo.

Material y métodos: Se realiza un estudio observacional de las reconstrucciones con injerto de tejido graso libre abdominal en pacientes que precisaron la realización de una parotidectomía (superficial, total o radical) debida a una neoplasia parotídea primaria o a una recidiva tumoral. Los pacientes fueron intervenidos por el mismo cirujano entre mayo de 2013 y enero de 2019. El estudio analiza aspectos epidemiológicos y clínicos de los pacientes, los abordajes y el tiempo quirúrgico, la estancia hospitalaria, las complicaciones postquirúrgicas, las secuelas, el seguimiento clínico y el resultado estético de los pacientes.

Resultados: Un total de 22 pacientes fueron intervenidos con utilización de injertos de grasa abdominal tras una parotidectomía superficial conservadora, total o radical. Fueron trece hombres y nueve mujeres de edades entre 13 y 79 años (46 años de media), con el diagnóstico inicial realizado mediante punción aspiración por aguja fina (PAAF) y tomografía computerizada (TC) o resonancia magnética (RM). El resultado estético en todos los paciente fue muy bueno o excelente. No secuelas a largo plazo ni síndrome de Frey. No complicaciones de interés, salvo leves hematomas y seromas postquirúrgicos.

Este trabajo fue presentado parcialmente en el XV Congreso Nacional de la Sociedad Española de Cirugía Oral y Maxilofacial celebrado en junio de 2019 en Sevilla.

\footnotetext{
${ }^{*}$ Autor para correspondencia:

Correo electrónico: drarribas@yahoo.es (Ignacio Arribas García).

DOI: 10.20986/recom.2020.1101/2019
}

1130-0558/@ 2020 SECOM. Publicado por Inspira Network. Este es un artículo Open Access bajo la licencia CC BY-NC-ND (http:// creativecommons.org/licenses/by-nc-nd/4.0/). 
Conclusión: Se presenta una técnica muy sencilla y con muy pocas complicaciones para la reconstrucción primaria de defectos postparotidectomía a través de mínimas incisiones con unos excelentes resultados estéticos.

\section{Reconstruction of parotid defects with abdominal free fat grafts. Clinical study of a simple surgical technique}

\section{A B S T R A C T}

Keywords:

Parotid gland, parotidectomy, parotid reconstruction, fat graft, facial aesthetics, facial reconstructive surgery, facial filling, parotid neoplasms.

\begin{abstract}
Introduction: Parotid surgery is a common technique with multiple approaches and reconstruction options. Primary reconstruction options are the superficial musculoaponeurotic system (SMAS) flap, the sternocleidomastoid muscle flap, free or microvascular grafts and biomaterials (Alloderm), among others.

We present our experience in periumbilical abdominal free fat graft for primary reconstruction of the parotid defect.

Material and methods: A observational study was performed in patients requiring a parotidectomy (superficial, total or radical) due to a primary parotid neoplasm or tumor recurrence. In all cases were used a free abdominal fat graft. The patients were operated by the same surgeon between May 2013 and January 2019. The study analyzes epidemiological and clinical aspects, approach, surgical time, hospital stay, post-surgical complications, sequelae, clinical follow-up and aesthetic results.

Results: A total of 22 patients were incluyed after a conservative, total or radical superficial parotidectomy. Thirteen men and nine women aged between 13 and 79 years (46 years on average), with the initial diagnosis made by fine water aspiration puncture (FNA) and Computerized Tomography (CT) or Magnetic Resonance Imaging (MRI). Aesthetic result in all patients was excellent or very good. No long-term sequelae or syndromes Frey. No complications interest except minor postsurgical hematomas and seroma.

Conclusion: A very simple technique with few complications is presented for the primary reconstruction of postparotidectomy defects with excellent aesthetic results.
\end{abstract}

\section{INTRODUCCIÓN}

La cirugía de la glándula parótida es una técnica muy habitual hoy en día y existen múltiples técnicas, abordajes y métodos de reconstrucción en función de cada centro hospitalario, escuela o especialidad. La parotidectomía superficial conservadora es, en general, el procedimiento estándar para los tumores del lóbulo superficial y la parotidectomía total y/o radical es la técnica habitualmente utilizada en tumores del lóbulo profundo y en tumores malignos con afectación del nervio facial. Las parotidectomías parciales se reservan por lo general para tumoraciones benignas localizadas en los extremos de la glándula.

La primera cirugía parotídea fue descrita en el año 160 d.C. ${ }^{1}$. En la época moderna, en 1823, Bernard describió el primer caso de un paciente intervenido de la parótida por un tumor de 8 años de evolución. Cadreanu, en 1892, realizó la primera parotidectomía describiendo la preservación del nervio facial ${ }^{2,3}$ y fue Avelino Gutiérrez quien describió en 1903 un abordaje específico para el acceso de la región parotídea ${ }^{4}$. No obstante, fue Blair, en 1912, y la posterior modificación de Bailey, en 1942, quienes describieron la incisión más utilizada para el abordaje del área parotídea ${ }^{5}$. Con el objetivo de mejorar la estética, fue Appiani en 1967 quien describió la utilización del abordaje de ritidectomía (o lifting modificada) para ocultar la cicatriz retroauricular en el cuero cabelludo y obtener mejores resultados estéticos ${ }^{6}$.

En la literatura hay publicados numerosos trabajos sobre la técnica quirúrgica, refinamientos técnicos y resultados sobre largas series de casos donde se describen las distintas entidades benignas y malignas, protocolos para mejorar la técnica y reducir las posibles complicaciones de esta delicada cirugía?.

Aparte de la cicatriz de la incisión, las dos principales secuelas que presentan los pacientes intervenidos de un tumor o alteración parotídea, excluyendo la lesión del nervio facial, son la deformidad o hundimiento de la región y la aparición del síndrome de Frey o síndrome auriculotemporal. El síndrome de Frey fue descrito por Lucja Frey en 1923, pero la observación de estos signos y síntomas se remonta al año 1740 por Kastremsky, a Duphenix en 1757 o a Baillanger en $1853^{8}$.

La deformidad o hundimiento de la región parotídea ha sido objeto de numerosos trabajos con muy diversas técnicas: colgajo de músculo esternocleidomastoideo (ECM) ${ }^{9-11}$, colgajo del sistema músculo aponeurótico superficial (SMAS) ${ }^{12-15}$, matrices dérmicas acelulares (Alloderm ${ }^{\mathrm{TM}}$, Dermamatrix $\left.{ }^{\mathrm{TM}}\right)^{16-19}$, platisma, entre otros con distintos resultados a largo plazo.

Se presenta la técnica que utiliza un injerto de tejido graso para la reconstrucción parotídea del defecto, un procedimien- 
to poco utilizado en el momento actual, que fue descrito por Walter en 1975 con el fin de evitar el hundimiento y aparición de síndrome de Frey ${ }^{20}$.

\section{Técnica quirúrgica}

Se coloca al paciente de cúbito supino y se preparan de manera simultánea los dos campos quirúrgicos: la región parotídea y la región centro abdominal. Se diseña la incisión parotídea preauricular y retroauricular en forma de "U" aprovechando arrugas faciales o utilizando la incisión previa en caso de una recidiva (Figura 1A). No utilizamos el abordaje de Avelino Gutiérrez ni el de ritidectomía en parotidectomías superficiales, solo en casos de parotidectomías complejas (totales con afectación nervio facial) o reintervenciones usando cicatriz previa. Se infiltra la incisión y la región subcutánea de la región parotídea con $10 \mathrm{ml}$ de Svedocain ${ }^{\circledR}$ 0,25 \% (bupivacaína 25 mg; epinefrina $50 \mu \mathrm{g}$ ). Se realiza la parotidectomía conservadora del nervio facial y nervio auricular mayor con la ayuda de unas tijeras Harmonic Focus ${ }^{\circledR}{ }^{\circledR}$ (Ethicon, Cincinnati, OH, EE. UU.), un dispositivo que combina una tijera disectora y un sellante de vasos/hemostático. Se completa una cuidadosa hemostasia y se realiza un abundante lavado de la región con suero salino templado (Figuras 1B y 1C). Para la obtención del injerto graso se infiltra de nuevo con $10 \mathrm{ml}$ de Svedocain ${ }^{\circledR} 0,25 \%$, se realiza pequeña incisión de $2-3 \mathrm{~cm}$ dentro de la región umbilical inferior para ocultar la cicatriz. Se realiza disección del tejido graso y se obtiene la cantidad adecuada en función del defecto a reconstruir, con leve sobrecorrección del defecto (Figura 1D). Se realiza una adecuada hemostasia y lavado del lecho quirúrgico y se coloca un hemostático en todo el lecho adiposo tipo Surgicel $^{\circledast}$ Original (hasta 2016) y Fibrillar (desde 2016) (Ethicon, Cincinnati, OH, EE. UU.) con vendaje compresivo y faja abdominal. El injerto graso se coloca inmediatamente en la zona del defecto sin apenas manipulación ni lavados para evitar contaminación o daño tisular (Figura 1E) y se sutura con Vicryl 3/0 Plus (Ethicon, Cincinnati, OH, EE. UU.) para evitar su migración. Se realiza cierre de las dos incisiones por planos y no se coloca ningún tipo de drenaje, solo apósitos compresivos en la región parotídea.

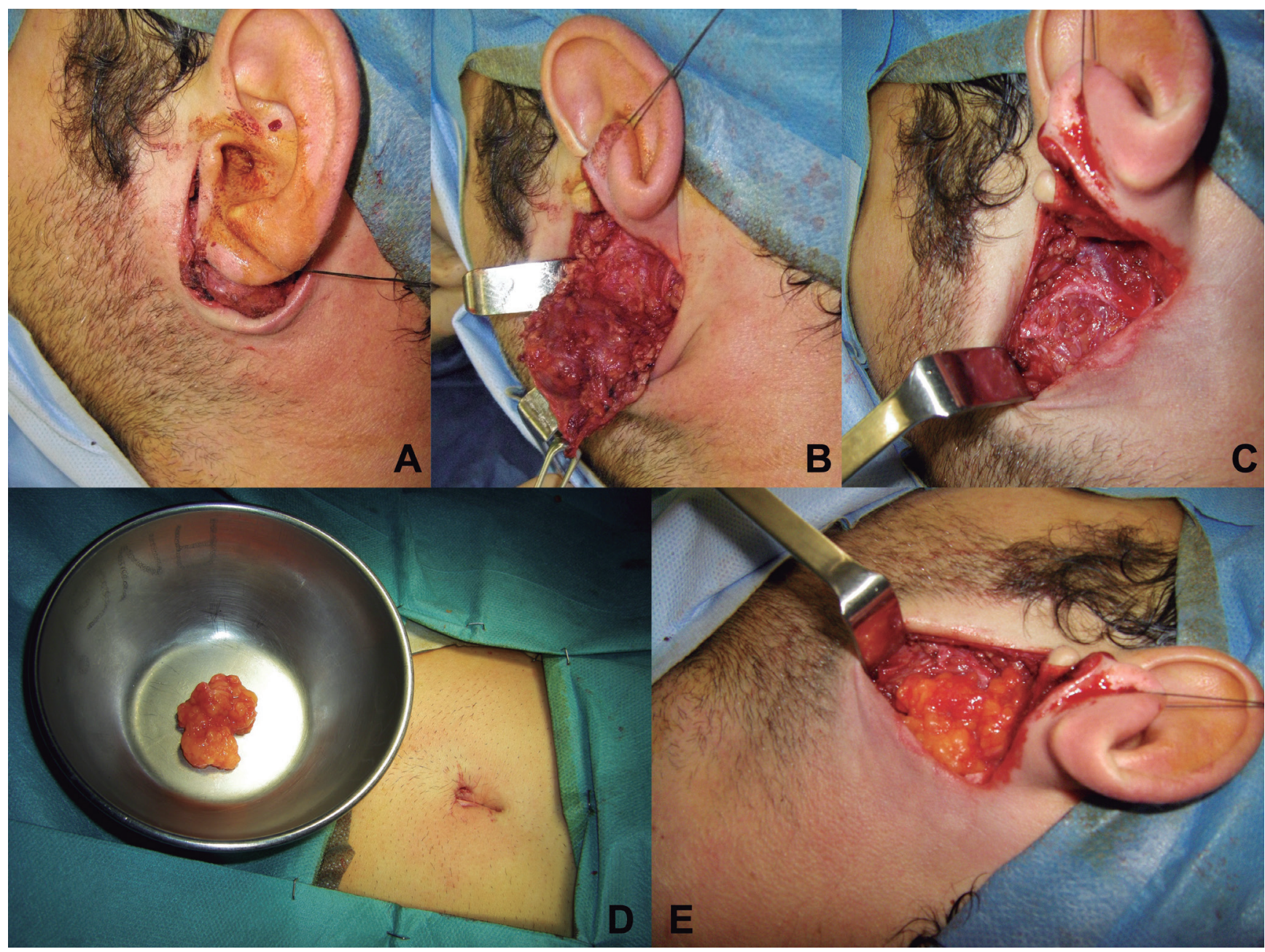

Figura 1. Técnica quirúrgica. A: incisión pre y postauricular en "U”. B: parotidectomía superficial conservadora de VII par.

C: lecho quirúrgico donde se observan ramas del nervio facial y el defecto de volumen. D: obtención de injerto de grasa libre abdominal a través de mínima incisión periumbilical inferior. E: colocación inmediata del injerto de grasa abdominal en el lecho quirúrgico tras su obtención. 


\section{MATERIAL Y MÉTODOS}

Se realiza un estudio observacional de pacientes que precisaron una parotidectomía (superficial, parcial, total o radical) debido a un tumor y se realizó una reconstrucción primaria de un defecto mediante un injerto de grasa libre abdominal. Los pacientes fueron intervenidos por el mismo cirujano y en el mismo Centro Hospitalario entre mayo de 2013 y enero 2019. Se analizan los datos de manera descriptiva y se incluyeron: edad, sexo, diagnóstico del paciente, tipo de abordaje, tiempo quirúrgico, estancia hospitalaria, complicaciones postquirúrgicas, secuelas, seguimiento clínico y resultado estético de los pacientes. Se propuso la técnica a todos los pacientes que precisaron una técnica de reconstrucción. No se incluyeron en este estudio pacientes que no necesitaron reconstrucción, pacientes de más de 80 años o pacientes que rechazaron la técnica propuesta. El estudio observacional se realizó siguiendo los principios de la Declaración de Helsinki.

\section{RESULTADOS}

Un total de 22 pacientes fueron incluidos en este estudio, 13 hombres y 9 mujeres, de edades entre 13 y 79 años con 46 años de media. Diecinueve pacientes presentaron un tumor primario parotídeo, dos pacientes desarrollaron una recidiva tumoral que fueron remitidas de otro Centro Hospitalario y un paciente presentó una metástasis tumoral. El diagnóstico inicial se realizó mediante una adecuada historia clínica y exploración física. El estudio se completó en todos los casos mediante punción aspiración con aguja fina guiada por ecografía (ECO-PAAF) y una prueba de imagen: el 35 \% (8) tomografía computerizada (TC) y el 65 \% (14) resonancia magnética (RM).

Los diagnósticos prequirúrgicos según ECO-PAAF y los diagnósticos anatomopatológicos definitivos se puede ver en la Tabla I.

En total se realizaron 23 parotidectomías (un paciente precisó parotidectomía bilateral por un tumor de Whartin bilateral): 21 parotidectomías fueron primarias y 2 parotidectomías fueron recidivas. De todas ellas, dos parotidectomías fueron totales en los pacientes que presentaron la recidiva tumo-

\begin{tabular}{|c|c|c|c|c|c|}
\hline ECO-PAAF & $\mathrm{n}$ & $\%$ & $\begin{array}{c}\text { Anatomía } \\
\text { patológica definitiva }\end{array}$ & $\mathrm{n}$ & $\%$ \\
\hline A pleomorfo & 10 & $43,5 \%$ & A pleomorfo & 10 & $45,5 \%$ \\
\hline T. Whartin & 6 & $26,1 \%$ & T. Whartin & 6 & $27,3 \%$ \\
\hline Melanoma & 1 & $4,3 \%$ & Melanoma & 1 & $4,5 \%$ \\
\hline \multirow[t]{5}{*}{$\begin{array}{l}\text { No } \\
\text { determinante }\end{array}$} & 6 & $26,1 \%$ & Cistoadenoma & 1 & $4,5 \%$ \\
\hline & & & Linfoadenoma & 1 & $4,5 \%$ \\
\hline & & & Linfoma tipo B & 1 & $4,5 \%$ \\
\hline & & & Mioepitelioma & 1 & $4,5 \%$ \\
\hline & & & Adenopatía & 1 & $4,5 \%$ \\
\hline
\end{tabular}

ral, una parotidectomía radical en el paciente que presentó metástasis de melanoma escleral y el resto de las veintiún parotidectomías fueron superficiales/parciales. El tipo de abordaje quirúrgico en todos los pacientes fue una incisión en " $U$ " pre y postauricular, salvo en los casos de las parotidectomías totales, donde se aprovechó el abordaje de Avelino Gutiérrez realizado previamente en dos pacientes y en otro se realizó abordaje tipo ritidectomía. En todos los casos se realizó injerto graso libre abdominal para relleno del defecto con el fin de mejorar la estética y evitar el síndrome de Frey.

El tiempo quirúrgico fue de 153 min de media (85-315); si excluimos las parotidectomías totales y radicales, y promediamos el caso bilateral, el tiempo medio fue de $132 \mathrm{~min}$ (85-210). La estancia media hospitalaria postquirúrgica fue de 1,52 días (1-8); solo dos pacientes estuvieron ingresados más de un día, 8 días en un paciente que precisó una parotidectomía total y presentaba un trastorno psiquiátrico asociado/problema social y 4 días en el paciente que presentó la metástasis por melanoma. El resto de pacientes fueron dados de alta al día siguiente de la intervención con estrecho seguimiento en consultas externas. El seguimiento medio fue de 30,6 meses (1077). Respecto a las complicaciones, 8 pacientes presentaron una paresia autolimitada de una de las ramas del nervio facial de menos de 2 meses de duración. Solo un paciente presentó parálisis completa debido a la parotidectomía radical realizada y actualmente solo presenta secuelas permanentes en las ramas orbitaria y frontal, las ramas marginales y bucales se realizó una reconstrucción con injerto de nervio auricular mayor en el mismo acto quirúrgico. Cinco pacientes desarrollaron un seroma autolimitado en lecho quirúrgico que precisaron de punción para su drenaje de manera ambulante. El resto de las complicaciones tipo inflamación, hematoma, etc., no fueron significativas.

En la Tabla II se resumen los datos de cada paciente y sus complicaciones y secuelas.

Se realizó encuesta de satisfacción estética a los pacientes a partir del sexto mes postquirúrgico. La pregunta realizada al paciente fue sobre su grado de satisfacción a nivel estético y los valores propuestos fueron entre: nada satisfecho, poco satisfecho, satisfecho, muy satisfecho y extremadamente satisfecho. El resultado fue: un paciente satisfecho (el paciente de metástasis por melanoma con parálisis facial postquirúrgica), 6 pacientes muy satisfechos y 15 pacientes extremadamente satisfechos, por lo que se puede afirmar que el resultado estético fue entre muy bueno y excelente. No se ha observado de forma clínica una atrofia especialmente significativa de los injertos grasos en el seguimiento de los pacientes.

No hemos registrado ningún síndrome de Frey en los pacientes ni secuelas zona donante.

\section{DISCUSIÓN}

La cirugía parotídea es una de las cirugías que más preocupación genera en el cirujano debido a las potenciales complicaciones y secuelas que se pueden originar en el paciente. La principal secuela sin duda es la posibilidad de lesión permanente del nervio facial y la consecuente parálisis facial, pero las secuelas estéticas debidas a la cicatriz y al hundimiento/ 


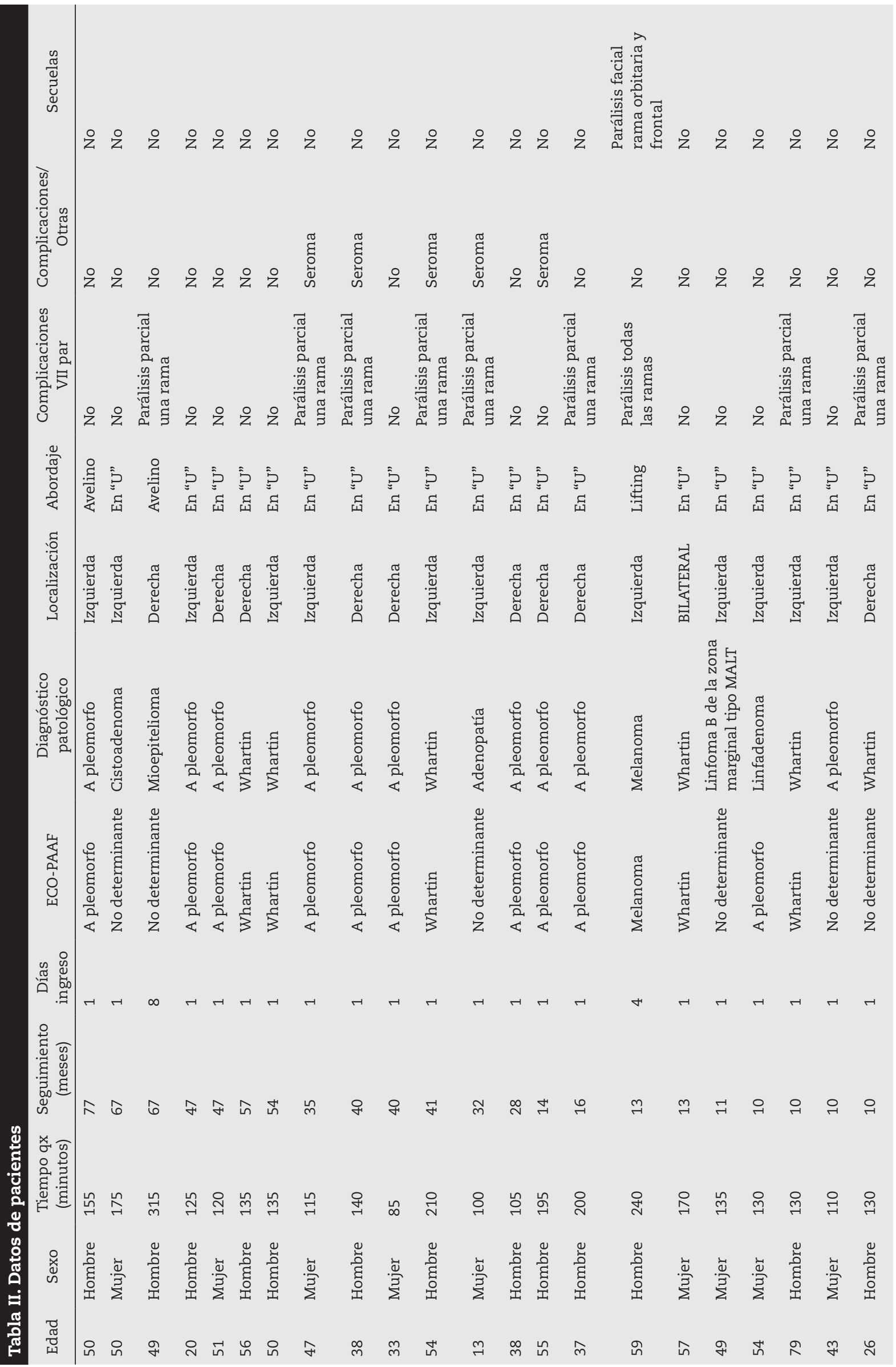


deformidad de la región parotídea son también fundamentales, sobre todo en los pacientes más jóvenes (Figura 2).

La utilización de incisiones cada vez más pequeñas y escondidas en los pliegues faciales es un gran avance en este tipo de cirugías. La incisión en "U" o mini lifting respetando el nervio auricular es la incisión que menos impacto estético tiene sobre la región parotídea y debe convertirse en nuestro gold-standard de cirugía parotídea no compleja. La utilización primaria de abordajes ampliados tipo Avelino Gutiérrez, Blair, Redon, Bailey, etc., debería reservarse a casos seleccionados por su complejidad o malignidad ${ }^{13}$.

La realización de reconstrucción primaria de los defectos parotídeos mediante diversas técnicas, como el colgajo de ECM, la disección y tracción de un colgajo de SMAS, en muchos casos son suficientes para obtener un resultado estético aceptable cuando la parotidectomía ha sido superficial o parcial. El hundimiento progresivo cuando se utiliza el colgajo de SMAS por su adaptación al defecto anatómico o la atrofia muscular del ECM cuando ha perdido su inervación hacen que se genere una asimetría facial leve en el paciente, que en muchos casos es evidente ${ }^{13,14}$.

La utilización de colgajos microvascularizados es una muy buena opción reconstructiva, sobre todo en grandes defectos oncológicos, donde es necesario grandes resecciones para poder obtener bordes quirúrgicos libres. En estos casos la aportación de tejido vivo es fundamental para la adecuada reconstrucción del paciente y es la mejor opción quirúrgica. Pero cuando el defecto no es tan grande o crítico, la utilización de los colgajos microvascularizados es cuestionable debido al riesgo y tiempo quirúrgico necesario, instrumental, personal y defecto de la zona donante, entre otras.

La utilización de materiales sintéticos o alodérmicos tipo Alloderm $^{\mathrm{TM}}$ o Dermamatrix ${ }^{\mathrm{TM}}$ pueden ser una opción válida en determinados defectos leves, pero su coste, su disponibilidad y sus posibles complicaciones tipo infección/rechazo/reacción cuerpo extraño hacen que sea una técnica que, en nuestra opinión, deber ser utilizada como segunda opción ${ }^{16-9,21}$.

El uso sistemático de injertos libres de grasa para la reconstrucción de los defectos parotídeos, ya sean de origen abdominal, inguinal o glútea, son una excelente opción reconstructiva. Es una técnica muy simple con excelentes resultados estéticos y sin apenas complicaciones o secuelas en el lecho quirúrgico o área donante. En la literatura hay diversos trabajos que utilizan esta técnica desde hace años con resultados similares a los obtenidos en este estudio. Balasundaram y cols. presentan 170 pacientes donde se empleó esta técnica con muy buenos
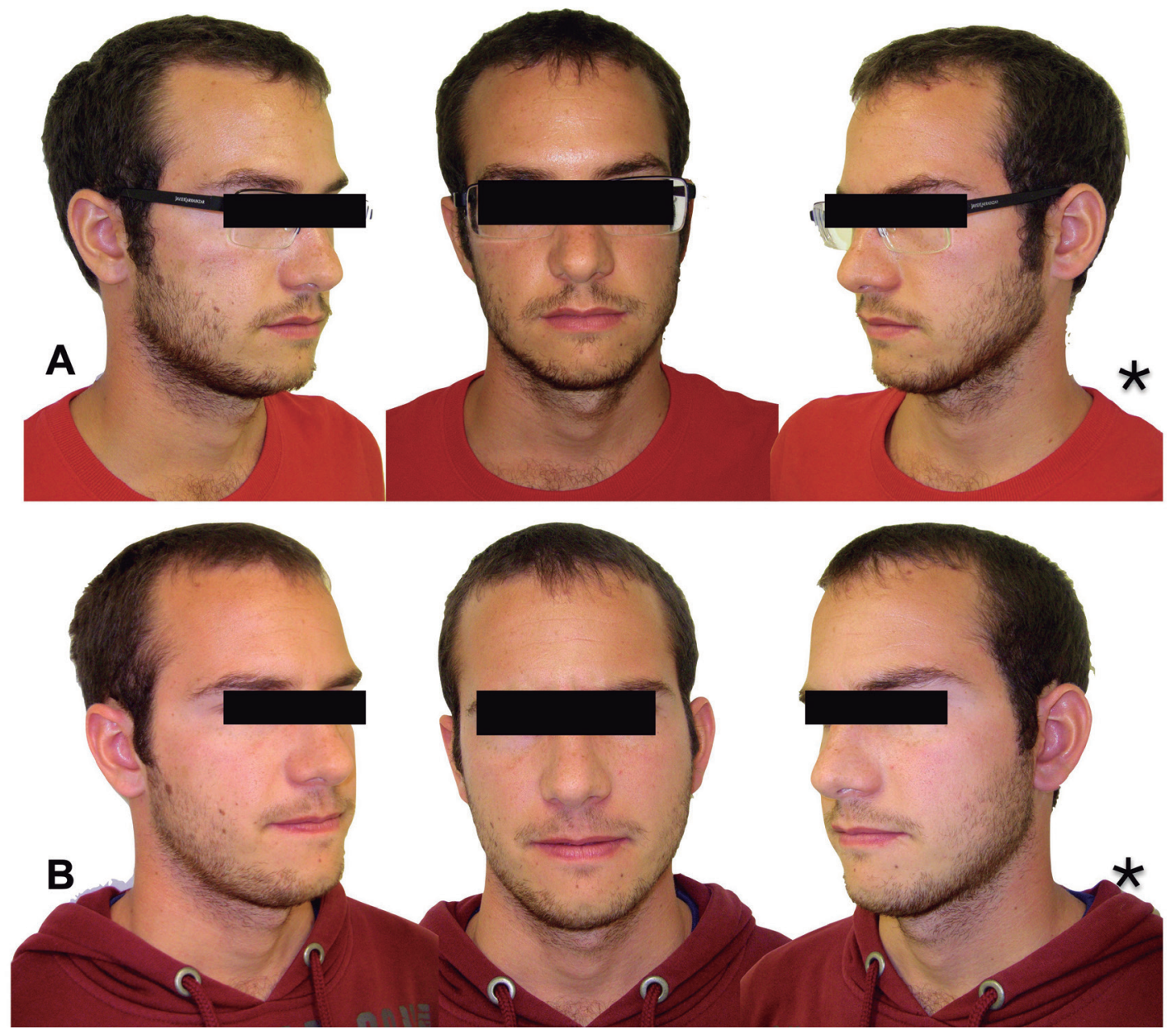

Figura 2. Imágenes del paciente de la Figura 1. 2A: imágenes preoperatorias del paciente y se señala con * el lado de afectación tumoral, en este caso lado izquierdo. 2B: imágenes postoperatorias a los 12 meses de la intervención. 
resultados estéticos y sin apenas desarrollo de complicaciones o síndrome de Frey. Refiere que el tejido adiposo paraumbilical es un sustituto ideal, muy parecido al tejido parotídeo y que apenas añade 15 minutos de tiempo quirúrgico, sin apenas morbilidad en región donante ${ }^{22}$. Honebrook y cols. utilizan injertos dermograsos suprapúbicos para reconstrucción defectos complejos craneofaciales, de los cuales 22 casos son defectos parotídeos con tan solo un caso de síndrome de Frey y unos resultados estéticos muy buenos: 4,5 de media en una escala de 1 al $5^{23}$. Otros autores con menor casuística comparten resultados similares, tanto a nivel estético como en reducción de complicaciones ${ }^{24,25}$. En nuestro estudio hemos optado por la utilización de grasa periumbilical debido al fácil acceso, al abundante tejido disponible y a las escasas complicaciones o secuelas de dicho abordaje. Esta técnica siempre se debe realizar a través de una mínima incisión y realizar una adecuada hemostasia con colocación de hemostáticos en lecho quirúrgico y ulterior vendaje compresivo. Los pacientes no refieren quejas de este abordaje tras el postoperatorio inmediato, tan solo la pena de no haber tenido que obtener mucho más tejido graso y haber realizado una liposucción completa y exhaustiva de toda la grasa abdominal (Figuras 2 y 3 ).

Si comparamos las técnicas reconstructivas más utilizadas, el colgajo de SMAS es una técnica que en ocasiones no es posible realizarla por la dificultad de disección del colgajo, la cercanía del tumor al SMAS o por el desgarro del mismo. Además, en nuestra opinión para grandes defectos, no siempre proporciona el adecuado volumen para un óptimo resultado estético y se desarrollan más síndromes de Frey. Respecto al colgajo de ECM, es una técnica sencilla pero limitada debido a varios motivos según nuestra experiencia. La primera limitación es que no aporta gran volumen si queremos reconstruir grandes defectos. Es un colgajo donde se produce mucha atrofia postquirúrgica y presenta un postoperatorio más doloroso que las otras técnicas expuestas. Además, genera en muchas ocasiones un defecto de la zona donante, que consiste en un defecto/hundimiento en la zona superior del ECM cercana a su inserción en la apófisis mastoides.

La reabsorción del injerto graso en nuestro estudio fue medido de manera clínica y no se apreció una gran reabsorción en ningún caso, tan solo alguna leve reabsorción del mismo. Por dicha apreciación clínica y según la opinión de los otros autores mencionados ${ }^{22,23}$ se recomienda una leve sobrecorrección volumétrica del defecto para compensar los efectos de una posible atrofia del injerto de tejido graso. Sería conveniente en un futuro poder realizar estudios para poder evaluar de manera más exacta y objetiva la reabsorción de este tipo de



Figura 3. A: imágenes preoperatorias del paciente y se señala con * el lado de afectación tumoral, en este caso lado derecho. B: imágenes postoperatorias a los 14 meses de la intervención. 
injertos con la utilización de técnicas de medición volumétricas basados en RM o TC.

La técnica reconstructiva de obtención del injerto graso y posterior adaptación al defecto quirúrgico incrementa levemente el tiempo quirúrgico si se compara a la opción de no reconstruir o utilización de otras técnicas locales. Este tiempo es difícil de cuantificar exactamente debido a que se utiliza otro campo y material quirúrgico, presencia de más o menos tejido graso en la región abdominal del paciente, presencia o no de sangrado del lecho quirúrgico, entre otros. Balasundaram y cols. lo estiman en 15 minutos aproximadamente ${ }^{22}$. La obtención del injerto graso no presenta apenas secuelas o complicaciones significativas y tiene una excelente aceptación por parte del paciente. Es, por tanto, una técnica sencilla con ventajas a nivel estético y supone la eliminación del síndrome Frey en todos los casos de nuestra serie.

\section{CONCLUSIÓN}

Se presenta un técnica sencilla de reconstrucción de defectos postparotidectomía con injerto de grasa libre abdominal periumbilical con un excelente resultado estético que apenas presenta complicaciones o secuelas en la zona parotídea o la zona donante abdominal.

\section{B I B L I O G R A F Í A}

1. RMR. Disease of the salivary glands. Filadelfia: WS Saunders; 2004. p. 138-9.

2. AM. History of the surgery of the facial nerve. Surgery of the facial nerve. Munchen: Urban \& Schwarzenberg; 1973.

3. Gûerrissi J. Cirugía de los tumores de la glándula parótida. Buenos Aires: Amolca; 2007. p. 18-9.

4. Avelino Gutiérrez. Tumores de la glandula parotida. Rev Cir. 1923;3:23-7.

5. Wormald R, Donnelly M, Timon C. 'Minor' morbidity after parotid surgery via the modified Blair incision. J Plast Reconstr Aesthetic Surg [Internet]. Elsevier; 2009;62(8):1008-11. DOI: 10.1016/j.bjps.2008.03.017.

6. AA. Surgical management of parotid tumours. Rev Argentina Cirugia. 1967;(21):236.

7. Irvine LE, Larian B, Azizzadeh B. Locoregional Parotid Reconstruction. Otolaryngol Clin North Am. 2016;49(2):435-46. DOI: 10.1016/j.otc.2015.10.012.

8. Dunbar EM, Singer TW, Singer K, Knight H, Lanska D, Okun MS. Understanding gustatory sweating. What have we learned from Lucja Frey and her predecessors? Clin Auton Res. 2002;12(3):179-84. DOI: 10.1007/s10286-002-0045-7.

9. Grosheva M, Horstmann L, Volk GF, Holler C, Ludwig L, Weiss $\mathrm{V}$, et al. Frey's syndrome after superficial parotidectomy: role of the sternocleidomastoid muscle flap: a prospective nonrandomized controlled trial. Am J Surg. 2016;212(4):740-7. DOI: 10.1016/j.amjsurg.2016.01.020.

10. Kerawala CJ, McAloney N, Stassen LFA. Prospective randomised trial of the benefits of a sternocleidomastoid flap after super- ficial parotidectomy. Br J Oral Maxillofac Surg. 2002;40(6):46872. DOI: 10.1016/S0266-4356(02)00194-8.

11. Gooden EA, Gullane PJ, Irish J, Katz M, Carroll C. Role of the sternocleidomastoid muscle flap preventing Frey's syndrome and maintaining facial contour following superficial pa-rotidectomy. J Otolaryngol. Canada; 2001;30(2):98-101. DOI: 10.2310/7070.2001.19876.

12. Rappaport I, Allison GR. Superficial musculoaponeurotic system amelioration of parotidec-tomy defects. Ann Plast Surg. 1985;14(4):315-23. DOI: 10.1097/00000637-198504000-00004.

13. Arden RL, Miguel GS. Aesthetic parotid surgery: evolution of a technique. Laryngoscope. 2011;121(12):2581-5. DOI: 10.1002/ lary.22154.

14. Kim I-K, Cho H-W, Cho H-Y, Seo J-H, Lee D-H, Park S-H. Facelift incision and superficial mus-culoaponeurotic system advancement in parotidectomy: case reports. Maxillofac Plast Re-constr Surg. 2015;37(1):40. DOI: 10.1186/s40902-0150040-2.

15. Manola M, Moscillo L, Simeon V, De Luca E, Mastella A. The Effectiveness of Sternocleido-mastoid Flap Versus Superficial Musculoaponeurotic System Flap for the Prevention of Frey Syndrome and Facial Depressed Deformity in Parotid Surgery for Pleomorphic Adenoma. Ann Plast Surg. 2018;80(2):125-9. DOI: 10.1097/SAP.0000000000001233.

16. Govindaraj S, Cohen M, Genden EM, Costantino PD, Urken ML. The use of acellular dermis in the prevention of Frey's syndrome. Laryngoscope. 2001;111(11 Pt 1):1993-8. DOI: 10.1097/00005537-200111000-00024.

17. Shridharani SM, Tufaro AP. A systematic review of acelluar dermal matrices in head and neck reconstruction. Plast Reconstr Surg. 2012;130(5 Suppl 2):35S-43S. DOI: 10.1097/ PRS.0b013e31825eff7a.

18. Athavale SM, Phillips S, Mangus B, Datta J, Sinard RJ, Netterville JL, et al. Complications of alloderm and dermamatrix for parotidectomy reconstruction. Head Neck. 2012;34(1):88-93. DOI: 10.1002/hed.21684.

19. Luo W, Zheng X, Chen L, Jing W, Tang W, Long J, et al. The use of human acellular dermal matrix in the prevention of infraauricular depressed deformities and Frey's syndrome fol-lowing total parotidectomy. Oral Surg Oral Med Oral Pathol Oral Radiol. 2012;114(2):e9-13. DOI: 10.1016/j.00oo.2011.08.012.

20. Walter $C$. The free dermis fat transplantation as adjunct in the surgery of the parotid gland (author's transl). Laryngol Rhinol Otol (Stuttg). 1975;54(5):435-40.

21. Sinha UK, Saadat D, Doherty CM, Rice DH. Use of AlloDerm implant to prevent frey syn-drome after parotidectomy. Arch Facial Plast Surg.2003;5(1):109-12.

22. Balasundaram I, Nasser NA. Paraumbilical fat graft for the correction of contour deformity following parotidectomy and prevention of Frey syndrome. Int J Oral Maxillofac Surg. 2016;45(3):380-2. DOI: 10.1016/j.ijom.2015.07.017.

23. Honeybrook A, Athavale SM, Rangarajan S V, Rohde SL, Netterville JL. Free dermal fat graft reconstruction of the head and neck: An alternate reconstructive option. Am J Otolaryngol. 2017;38(3):291-6. DOI: 10.1016/j.amjoto.2017.01.026.

24. Harada T, Inoue T, Harashina T, Hatoko M, Ueda K. Dermis-fat graft after parotidectomy to prevent Frey's syndrome and the concave deformity. Ann Plast Surg. 1993;31(5):450-2. DOI: 10.1097/00000637-199311000-00012.

25. Vico PG, Delange A, De Vooght A. Autologous fat transfer: an aesthetic and functional re-finement for parotidectomy. Surg Res Pract. 2014;2014:873453. 